\title{
Combined prostaglandin E1 and lithium exert potent neuroprotection in a rat model of cerebral ischemia
}

\author{
Rui SHENG\#, Li-sha ZHANG\#, Rong HAN, Bo GAO, Xiao-qian LIU, Zheng-hong QIN* \\ Department of Pharmacology and Laboratory of Aging and Nervous Diseases, Soochow University School of Medicine, Suzhou \\ 215123, China
}

\begin{abstract}
Aim: To examine the effects of a mixed formulation composed of prostaglandin E1 and lithium (PGE1+Li mixture) on brain damage after cerebral ischemia. The effects of the mixture on protein expression of heat shock proteins (HSPs), p53, and Bcl-2 were also determined.

Methods: Brain ischemia was induced with a permanent middle cerebral artery occlusion (pMCAO) in rats. Rats were treated with a single intravenous administration of PGE1, lithium or a PGE1+Li mixture immediately after the ischemic insult. The infarct volume and motor behavior deficits were analyzed $24 \mathrm{~h}$ after the ischemic insult. The protein levels of HSP70, glucose-regulated protein 78 (GRP78), HSP60, Bcl-2, and p53 in the striatum of the ipsilateral hemisphere were examined using immunoblotting.

Results: The mixture (PGE1 $22.6 \mathrm{nmol} / \mathrm{kg}+\mathrm{Li} 0.5 \mathrm{mmol} / \mathrm{kg}$ ) reduced infarct volume and neurological deficits induced by focal cerebral ischemia. Moreover, the mixture had a greater neuroprotective effect against cerebral ischemia compared with PGE1 or lithium alone. The mixture was effective even if it was administered $3 \mathrm{~h}$ after ischemia. PGE1+Li also significantly upregulated cytoprotective HSP70, GRP78, HSP60, and Bcl-2 protein levels, while decreasing p53 expression.

Conclusion: These results demonstrated a PGE1+Li mixture with a therapeutic window of up to $3 \mathrm{~h}$ for clinical treatment of cerebral ischemia. The PGE1+Li mixture potentially exerts a protective effect after stroke through the induction of HSPs and Bcl-2 proteins.
\end{abstract}

Keywords: prostaglandin E1; lithium; PGE1+Li mixture; heat shock proteins; cerebral ischemia; Bcl-2; p53

Acta Pharmacologica Sinica (2011) 32: 303-310; doi: 10.1038/aps.2010.211; published online 24 Jan 2011

\section{Introduction}

Development of neuroprotective agents against ischemiainduced brain damage is needed to reduce the mortality and morbidity associated with stroke ${ }^{[1]}$. Heat shock proteins (HSPs) are molecular chaperones that regulate folding of nascent and denatured proteins, act as transport proteins between subcellular compartments and modify the activities of proteins by altering their conformational states ${ }^{[2]}$. HSPs may exert neuroprotective effects by antagonizing apoptotic and necrotic cell death during cerebral ischemia ${ }^{[3]}$. Induction of HSPs also plays a role in the preconditioning-induced resistance of neurons to ischemic insults ${ }^{[4,5]}$. HSPs such as HSP70, glucose-regulated protein 78 (GRP78) and HSP60 are important regulators of cellular survival and may be used as potential therapeutic targets for treating ischemic neuronal injury ${ }^{[6-8]}$.

Prostaglandin E1 (PGE1) has several pharmacological

\footnotetext{
\# These authors contributed equally to this work.

* To whom correspondence should be addressed.

E-mail Zhqin5@hotmail.com

Received 2010-08-16 Accepted 2010-11-23
}

effects, including cytoprotection, vasodilation, inhibition of platelet aggregation, membrane stabilization and anti-inflammation $^{[9]}$. The clinical uses of PGE1 include the treatment of ischemic diseases such as cerebral, myocardial and hepatic ischemia. In recent studies, Matsuo reported that PGE1 induces HSP70, GRP78, and HSP86 immediately after hepatic ischemia reperfusion. Therefore, HSPs might play an important role in mediating the protective actions of PGE1 against ischemia/reperfusion injury in the liver ${ }^{[10]}$.

Lithium has been extensively used in the treatment of bipolar mood disorders ${ }^{[11]}$. Growing evidence suggests that lithium is a neuroprotective drug that is effective against a variety of insults, including glutamate-induced excitotoxicity, ischemia-induced neuronal damage and other neurodegenerative conditions $^{[12]}$. Recently, Ren et al ${ }^{[13]}$ reported that treatment of rats with lithium decreased the infarct volume in a permanent focal cerebral ischemia model, and the neuroprotective effects of lithium were associated with the upregulation of cytoprotective HSP70 in the ischemic brain hemispheres.

In a previous study, research from our laboratory ${ }^{[14]}$ found that lithium could potentiate the neuroprotective effects of PGE1 through synergistic induction of HSPs, but the differ- 
ent administration routes of PGE1 (intravenous injection, iv) and lithium (subcutaneous injection, sc) make the clinical administration of drug combination inconvenient. Therefore, in the present study, we formulated a PGE1+Li mixture for intravenous administration. We verified that the mixture had neuroprotective actions with a therapeutic window up to $3 \mathrm{~h}$. The mixture also induced the expression of HSPs and Bcl-2 in animals with ischemic injuries.

\section{Materials and methods} Preparation of the PGE1+Li mixture and experimental protocol PGE1 (NanYang Pukang Pharmaceutical Co ltd, Henan, China) and lithium (Sigma, St Louis, MO, USA) were dissolved successively in sterile normal saline ( $\mathrm{pH}$ 7.2-7.4) to prepare the PGE1+Li mixture. Male Sprague-Dawley rats weighing 280-300 g were purchased from the Center for Experimental Animals at Soochow University. The NIH guidelines for the Care and Use of Laboratory Animals were followed during all animal procedures. In this study, three batches of rats were used. The first batch of 70 rats was randomly divided into 7 groups: the sham-operated group, the permanent middle cerebral artery occlusion (pMCAO) group, the lithium group ( $\mathrm{Li} 0.5 \mathrm{mmol} / \mathrm{kg}$ ), the PGE1(S) group (PGE1 $22.6 \mathrm{nmol} / \mathrm{kg}$ ), the PGE1(L) group (PGE1 $45.2 \mathrm{nmol} / \mathrm{kg}$ ), the PGE1(S)+Li mixture group (PGE1 $22.6 \mathrm{nmol} / \mathrm{kg}+\mathrm{Li} 0.5$ $\mathrm{mmol} / \mathrm{kg}$ ), and the PGE1(L)+Li mixture group (PGE1 45.2 $\mathrm{nmol} / \mathrm{kg}+\mathrm{Li} 0.5 \mathrm{mmol} / \mathrm{kg}$ ). The regional cerebrocortical blood flow $(\mathrm{rCBF})$ in the rats was monitored, and the infarct volume, brain water content and neurological deficits were also evaluated in the animals. A second batch of 50 rats was randomly divided into 5 groups: the sham-operated group, the pMCAO group, the lithium group, the PGE1(S) group, and the PGE1(S)+Li mixture group. These rats were used for the immunoblotting procedures. In these two experiments, PGE1, lithium, and the PGE1+Li mixture were injected intravenously immediately after the onset of pMCAO. The last batch of 40 rats was randomly divided into 4 groups: the pMCAO group, the PGE1(S)+Li mixture $1.5 \mathrm{~h}$ group, the PGE1(S)+Li mixture $3 \mathrm{~h}$ group, and the PGE1(S)+Li mixture $6 \mathrm{~h}$ group. These rats were used to evaluate the therapeutic window of the PGE1+Li mixture for the treatment of ischemia. The PGE1(S)+Li mixture was administered $1.5 \mathrm{~h}, 3 \mathrm{~h}$, or $6 \mathrm{~h}$ after the onset of pMCAO. In all of the experiments, sham-operated and model animals received injections of normal saline using the same volume as the various treatments.

\section{Rat pMCAO model}

The rat pMCAO model was produced using the intraluminal suture technique described by Longa with minor modifications ${ }^{[15,16]}$. The rats were anesthetized with $4 \%$ chloral hydrate $(350 \mathrm{mg} / \mathrm{kg})$. A $30 \mathrm{~mm}$ length of monofilament nylon suture $(\Phi 0.22-\Phi 0.24 \mathrm{~mm})$ with a rounded tip was inserted into the internal carotid artery through a small incision in the right common carotid artery and then advanced to the Circle of Willis. The suture remained in place until the rats were killed. Laser-Doppler flowmetry (LDF, ML191 Laser Doppler Blood
FlowMeter) was used to monitor the blockade of cerebral blood flow. The rCBF was detected prior to onset of ischemia to acquire the preischemia blood flow level. After the pMCAO operation, the rCBF sharply dropped to approximately $5 \%-10 \%$ of the preischemia value. Then the rCBF was measured again 5, 10, 15, 30, 60, and 120 min after drug administration $^{[14]}$. Body temperature was closely monitored with a rectal probe and maintained in the range of $37.0 \pm 0.5^{\circ} \mathrm{C}$ with a heating pad (Institute of Biomedical Engineering, CAMS, BME-412A ANIMAL REGULATOR) during and after surgery until the animals recovered from the anesthesia. Shamoperated rats underwent the same procedures except for the pMCAO. About $20 \%-30 \%$ of the rats died $24 \mathrm{~h}$ after ischemia onset and were excluded from further analysis. Rats that showed tremors and seizures were also excluded from further analysis.

\section{Evaluation of infarct volume, brain water content and neuro- logical deficit}

Twenty-four hours after ischemia, the neurological deficits in rats subjected to PMCAO were evaluated with a protocol that was previously described by an observer who was blinded to the treatment ${ }^{[15]}$. A total score of a possible 10 was determined as follows: (1) when the rats were suspended by the tail, the left forelimb was flexed and the flex was scored 1-4 according to severity; (2) when rats were placed on a smooth plane, the lateral push resistance toward the left side decreased, which was scored from 1-3; (3) the rats were pulled gently backward by the tail and the left forelimb showed decreased strength, which was scored from 1-3. After the animals were scored, they were euthanized. The brains were dissected out and sliced in a plastic module (3-mm thickness, Harvard Apparatus, MA, USA). Then 5 slices of coronal sections were stained with $4 \%$ 2, 3, 5-triphenyltetrazolium chloride (TTC) for 30 min and fixed with $4 \%$ paraformaldehyde. Image analyzing software called SigmaScan Pro5 was used to measure brain infarcts. Considering the fact that infarct volume expands due to edematous change, the infarct volume was calculated with the following formula: infarct volume $=($ red area of the contralateral side - red area of the ipsilateral side)/total area $\times 100 \%{ }^{[17]}$.

\section{Immunoblotting}

Immunoblotting was carried out as previously described ${ }^{[18]}$. Brain tissues from the ischemic striatum of the right middle cerebral artery territory and the corresponding area of the sham-operated rats were homogenized. Then the proteins were extracted with a lysis buffer $(10 \mathrm{mmol} / \mathrm{L}$ Tris- $\mathrm{HCl}, \mathrm{pH}$ 7.4, $150 \mathrm{mmol} / \mathrm{L} \mathrm{NaCl}, 1 \%$ Triton-100, 0.1\% SDS, $5 \mathrm{mmol} / \mathrm{L}$ ethylenediaminetetraacetic acid [EDTA], $1 \mathrm{mmol} / \mathrm{L}$ phenylmethylsulfonyl fluoride [PMSF], $0.28 \mathrm{U} / \mathrm{mL}$ aprotinin, $50 \mu \mathrm{g} / \mathrm{mL}$ leupeptin, $1 \mathrm{mmol} / \mathrm{L}$ benzamidine, $7 \mu \mathrm{g} / \mathrm{mL}$ pepstatin A). The protein concentrations were determined (SmartSpec3000 Spectrophotometer, Bio-Rad, Hercules, CA, USA) using a BCA kit (Pierce, Rockford, IL, USA). A $50 \mu \mathrm{g}$ aliquot of protein from each sample was separated with a 10\% SDS-PAGE gel and subsequently transferred to a nitrocellulose membrane. 
Afterward, the membranes were incubated with antibodies against HSP70 (1:200; Mouse derived anti-HSP70 monoclonal antibody; Santa Cruz Biotechnology, Santa Cruz, CA, USA), GRP78 (1:400; Rabbit derived anti-GRP78 polyclonal antibody; Stressgen Bioreagents, Canada), HSP60 (1:100; Mouse derived anti-HSP60 monoclonal antibody; Sigma, St Louis, MO, USA), p53 (1:1000; Mouse derived anti-p53 monoclonal antibody; Cell Signaling, Woburn, MA, USA), or Bcl-2 (1:200; Rabbit derived anti-Bcl-2 polyclonal antibody; Santa Cruz Biotechnology, Santa Cruz, CA, USA) at $4^{\circ} \mathrm{C}$ for $3 \mathrm{~h}$. Next, the membranes were incubated with a horseradish peroxidaseconjugated secondary antibody (1:5000; Sigma, St Louis, MO, USA) at room temperature for $1 \mathrm{~h}$. Immunoreactivity was detected with enhanced chemiluminescent autoradiography (ECL kit; Amersham, Piscataway, NJ, USA) according to the manufacturer's instructions. The membranes were reprobed with $\beta$-actin (1:5000; Sigma) after the membrane was stripped with Tris-buffered saline containing $0.1 \%$ Tween-20 (TBST) and $2 \% \beta$-mercaptoethanol at $65^{\circ} \mathrm{C}$ for $1 \mathrm{~h}$.

\section{Statistical analysis}

Statistical analysis was carried out with one-way ANOVA. The intergroup comparisons (post-hoc analysis) among data with equal variances were carried out with the least significant difference (LSD) method, whereas Tamhane's T2 method was used for data with unequal variances. $P<0.05$ was considered significant.

\section{Results}

\section{PGE1+Li mixture had no significant improvement on rCBF}

After the pMCAO operation, the rCBF decreased to approximately $5 \%-10 \%$ of the preischemic values and remained low in all of the rats that were subjected to pMCAO. PGE1, lithium and the PGE1+Li mixture produced no statistically significant improvements in the reduction of $\mathrm{rCBF}$ within $5 \mathrm{~min}, 10 \mathrm{~min}$, $15 \mathrm{~min}, 30 \mathrm{~min}, 60 \mathrm{~min}$, and $120 \mathrm{~min}$ after the surgical operation (Table 1).

\section{PGE1+Li mixture reduced pMCAO-induced brain damage}

In the rats subjected to pMCAO for $24 \mathrm{~h}$, extensive infarction was detected in the cerebral cortical and subcortical areas over a series of brain sections. Treatment with a single intravenous injection of lithium $(0.5 \mathrm{mmol} / \mathrm{kg})$, PGE1 (S), PGE1(L), the PGE1(S)+Li mixture or the PGE1(L)+Li mixture immediately after the onset of pMCAO resulted in a significant reduction in infarct volume that was detected by TTC staining $(P<0.05$ or $P<0.01$ vs model group). Moreover, administration of the PGE1(S)+Li mixture (PGE1 $22.6 \mathrm{nmol} / \mathrm{kg}+\mathrm{Li} 0.5 \mathrm{mmol} / \mathrm{kg}$ ) produced a greater reduction in infarct volume $(P<0.01$ vs PGE1(S) group) (Figure 1A and 1B).

Rats subjected to pMCAO were examined and scored for motor deficits using a 10-point scale as described in the Methods. The pMCAO rats displayed marked motor behavioral deficits. Treatment with lithium, PGE1(S), PGE1(L), the PGE1(S)+Li mixture or the PGE1(L)+Li mixture resulted in a significant reduction in behavioral deficits $(P<0.05$ or $P<0.01$ vs model group). In addition, administration of the PGE1(S)+Li mixture produced a greater improvement in motor deficits $(P<0.05$ vs PGE1(S) group) (Figure $1 \mathrm{C})$.

\section{The therapeutic window of the PGE1+Li mixture's neuroprotecion on PMCAO}

We sought to determine the time interval after ischemia in which the PGE1+Li mixture would be able to protect the brain (therapeutic window). The PGE1(S)+Li mixture was administered $1.5,3$, or $6 \mathrm{~h}$ after the onset of pMCAO. Significant infarct volume reductions were observed when the PGE1+Li mixture was administered $1.5 \mathrm{~h}(-36.6 \%)$ or $3 \mathrm{~h}(-31.3 \%)$ after ischemia ( $P<0.01$ vs model group), but not when the administration of the mixture was delayed by $6 \mathrm{~h}(P>0.05$; Figure 2$)$.

\section{Enhanced induction of HSP70, HSP60, and GRP78 by the PGE1+Li mixture}

The expression of HSP70, GRP78 and HSP60 was significantly upregulated in the ischemic striatum after pMCAO $(P<0.05$ vs sham group). Although PGE1(S) $(22.6 \mathrm{nmol} / \mathrm{kg})$ or lithium $(0.5 \mathrm{mmol} / \mathrm{kg})$ alone had no significant effects on the these proteins, the PGE1(S)+Li mixture (PGE1 $22.6 \mathrm{nmol} / \mathrm{kg}+\mathrm{Li} 0.5$ $\mathrm{mmol} / \mathrm{kg}$ ) significantly increased HSP70, GRP78, and HSP60 protein levels compared with both the model group and the PGE1(S) group $(P<0.05$ or $P<0.01$ vs model group and PGE1(S) group, Figure 3-4).

Table 1. The effects of the PGE1+Li mixture on $\mathrm{rCBF}$ (\% of preischemia value) in rats subjected to pMCAO. The rCBF was measured in the ipsilateral ischemic hemisphere with LDF before the onset of ischemia, immediately after ischemia and 5, 10, 15, 30, 60, and 120 min after drug administration. Postischemic rCBF values are expressed as a percentage of the preischemic values (100\%). PGE1(S)=PGE1 $22.6 \mathrm{nmol} / \mathrm{kg}, \mathrm{PGE} 1(\mathrm{~L})=\mathrm{PGE} 1 \mathrm{45.2}$ nmol/kg. $n=6$ rats. The data are expressed as means \pm SD. ${ }^{c} P<0.01$ compared with preischemia.

\begin{tabular}{|c|c|c|c|c|c|c|c|}
\hline & \multicolumn{7}{|c|}{ Time after drug administration } \\
\hline & $0 \mathrm{~min}$ & $5 \mathrm{~min}$ & $10 \min$ & $15 \min$ & $30 \min$ & $1 \mathrm{~h}$ & $2 \mathrm{~h}$ \\
\hline Model & $9.6 \pm 6.5^{c}$ & $9.5 \pm 5.9$ & $9.4 \pm 6.4$ & $10.0 \pm 5.3$ & $10.1 \pm 5.2$ & $10.5 \pm 7.1$ & $9.8 \pm 6.4$ \\
\hline PGE1(S) & $8.1 \pm 6.0^{c}$ & $14.2 \pm 8.3$ & $14.8 \pm 7.6$ & $14.6 \pm 8.5$ & $13.3 \pm 6.9$ & $9.6 \pm 7.7$ & $9.4 \pm 6.7$ \\
\hline PGE1(L) & $10.2 \pm 6.2^{c}$ & $14.0 \pm 8.6$ & $15.2 \pm 6.5$ & $12.8 \pm 6.4$ & $12.3 \pm 8.5$ & $12.0 \pm 6.6$ & $11.0 \pm 7.4$ \\
\hline Lithium & $10.9 \pm 5.8^{c}$ & $11.3 \pm 8.1$ & $12.0 \pm 8.4$ & $11.1 \pm 8.6$ & $11.2 \pm 9.5$ & $11.1 \pm 5.8$ & $11.2 \pm 6.4$ \\
\hline
\end{tabular}




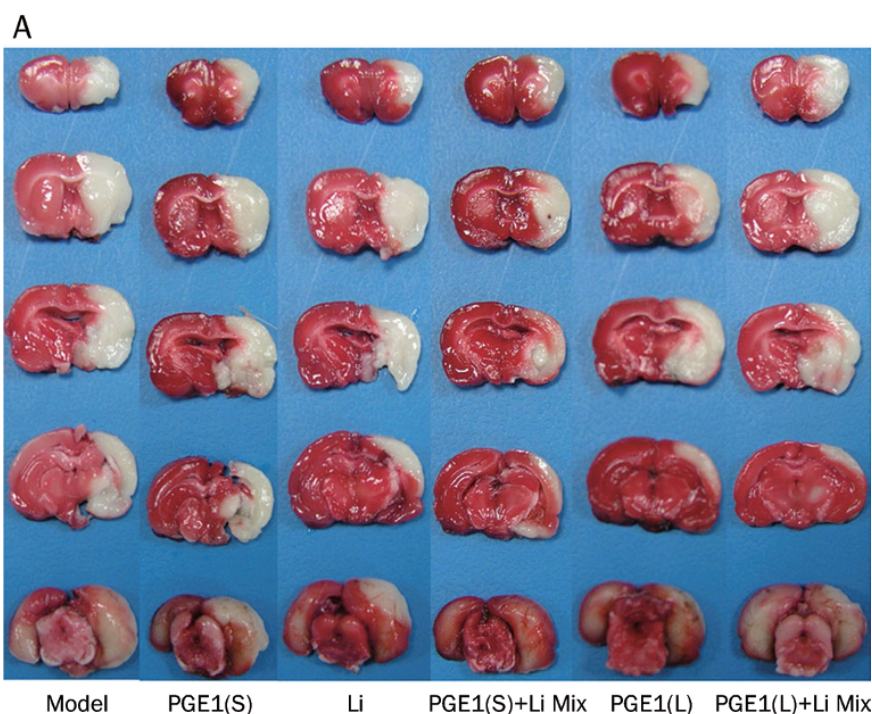

B

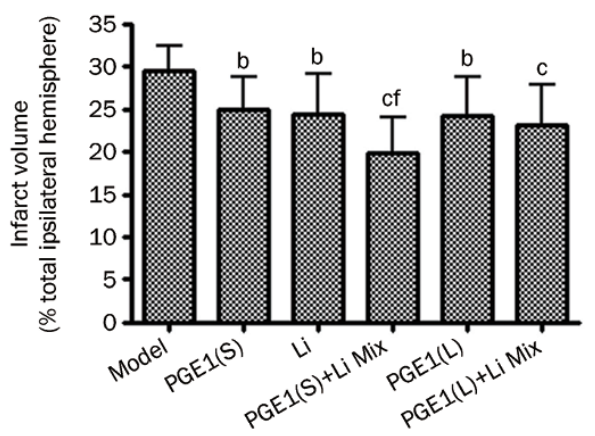

C

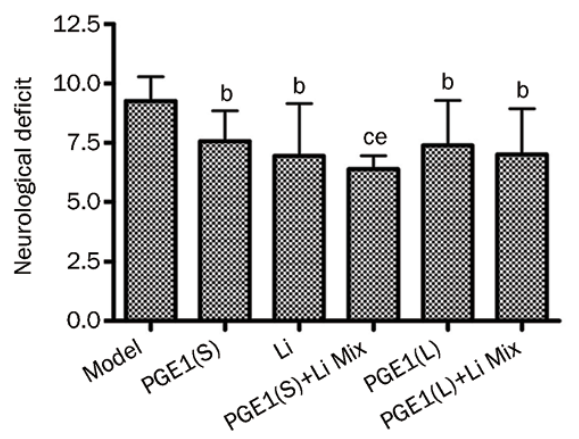

Figure 1. The PGE1+Li mixture reduced PMCAO-induced cerebral ischemia. Rats were injected intravenously with PGE1, Li and a PGE1+Li mixture immediately after PMCAO. The rats were euthanized $24 \mathrm{~h}$ after ischemia. (A) TTC staining of brain sections. The infarct brain regions are white. The results indicated that animals treated with PGE1, lithium or the PGE1(S)+Li mixture had smaller infarct volumes. (B) Quantitative analysis of brain infarct volume. (C) PGE1 and lithium treatment reduced pMCAO-induced neurological deficits. PGE1(S)=PGE1 $22.6 \mathrm{nmol} / \mathrm{kg}$, $P G E 1(L)=P G E 145.2 \mathrm{nmol} / \mathrm{kg} . n=6$ rats. The bar represents the mean \pm SD. ${ }^{\mathrm{b}} P<0.05,{ }^{\mathrm{c}} P<0.01$ compared with the model group; ${ }^{\mathrm{e}} P<0.05,{ }^{\mathrm{f}} P<0.01$ compared with the PGE1(S) group.

\section{A}
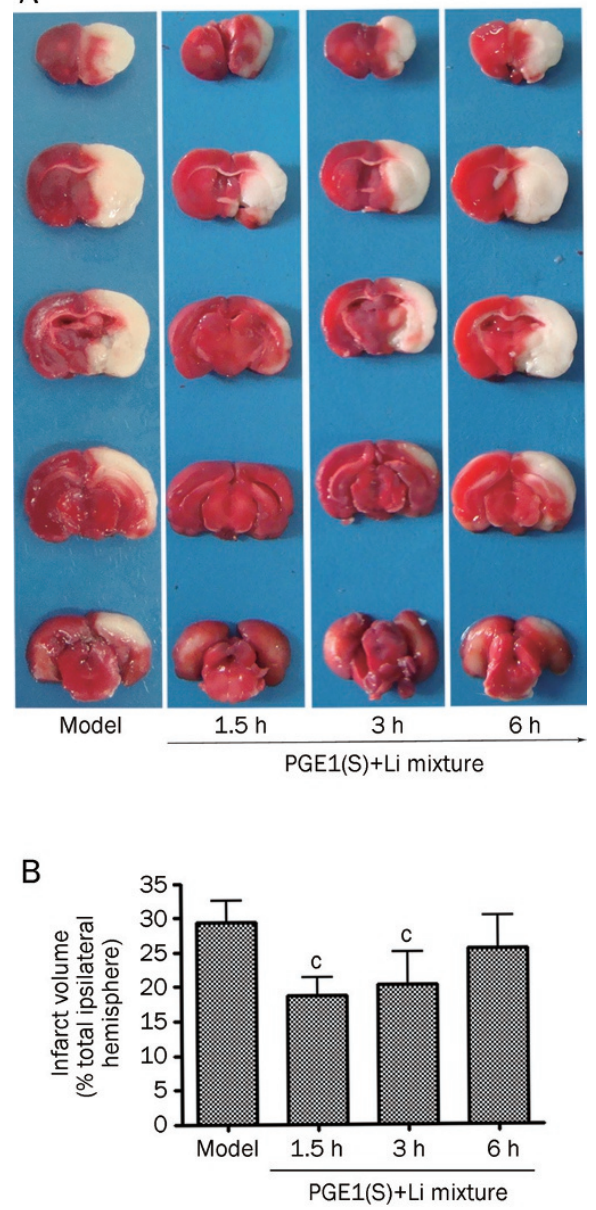

Figure 2. The therapeutic window of the neuroprotective effect of the PGE1+Li mixture in the pMCAO model. Rats were injected intravenously with the PGE1(S)+Li mixture (PGE1 $22.6 \mathrm{nmol} / \mathrm{kg}+\mathrm{Li} 0.5 \mathrm{mmol} / \mathrm{kg}$ ) $1.5,3$, or $6 \mathrm{~h}$ after the PMCAO. (A) TTC staining of brain sections. The infarct brain regions are white. (B) Quantitative analysis of brain infarct volume. PGE1(S)=PGE1 $22.6 \mathrm{nmol} / \mathrm{kg} . n=6$ rats. The bar represents the mean $\pm S D$. ${ }^{c} P<0.01$ compared with the model group.

PGE1+Li mixture increased Bcl-2, but decreased p53 protein levels

Expression of Bcl-2 was significantly downregulated in the ischemic striatum after pMCAO. Lithium significantly upregulated Bcl-2 protein levels compared with the model group $(P<0.01$ vs model group). Furthermore, the PGE1(S)+Li mixture (PGE1 $22.6 \mathrm{nmol} / \mathrm{kg}+\mathrm{Li} 0.5 \mathrm{mmol} / \mathrm{kg}$ ) further increased $\mathrm{Bcl}-2$ protein levels $(P<0.01$ vs model group and PGE1(S) group, Figure 5A).

Expression of p53 was significantly upregulated in the ischemic striatum after pMCAO. However, PGE1(S) (22.6 $\mathrm{nmol} / \mathrm{kg})$ or lithium $(0.5 \mathrm{mmol} / \mathrm{kg})$ significantly decreased p53 protein levels compared with the model group $(P<0.05$ or $P<0.01$ vs model group). In addition, the PGE1(S)+Li mixture (PGE1 $22.6 \mathrm{nmol} / \mathrm{kg}+\mathrm{Li} 0.5 \mathrm{mmol} / \mathrm{kg}$ ) further decreased p53 
A
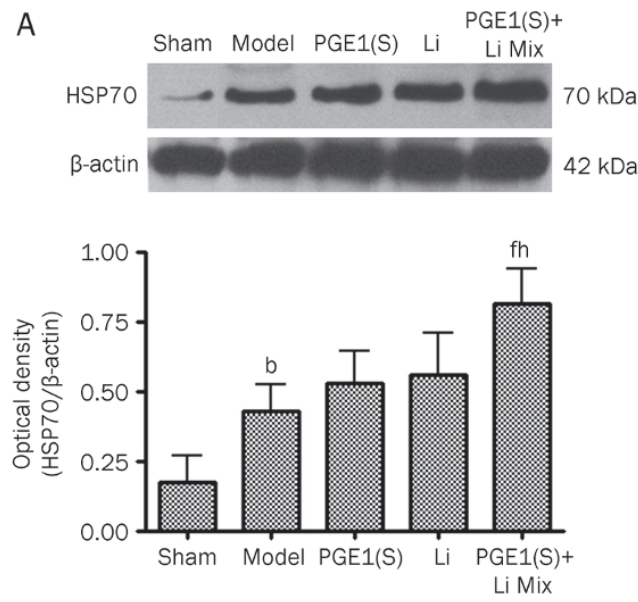

B
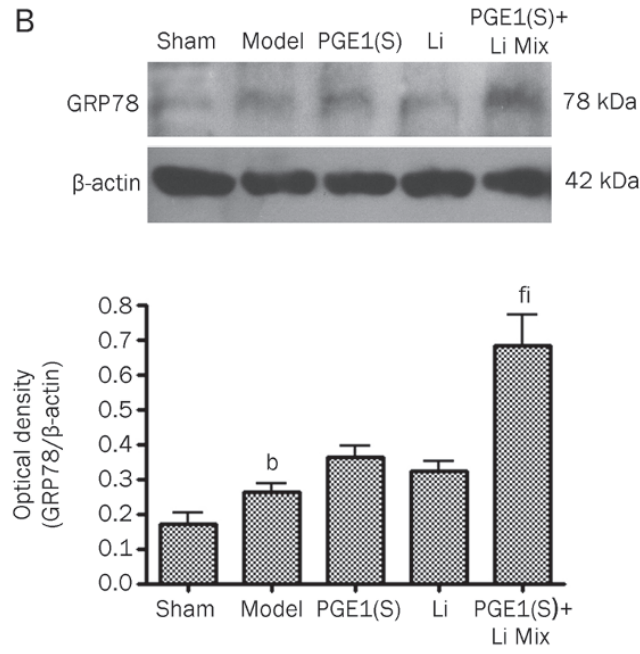

Figure 3. The PGE1+Li mixture enhanced PMCAO-induced HSP7O and GRP78 expression. The rats were injected intravenously with PGE1(S) $22.6 \mathrm{nmol} / \mathrm{kg}$, Li $0.5 \mathrm{mmol} / \mathrm{kg}$ or a PGE1(S)+Li mixture (PGE1 22.6 $\mathrm{nmol} / \mathrm{kg}+\mathrm{Li} 0.5 \mathrm{mmol} / \mathrm{kg}$ ) immediately after pMCAO. The rats were euthanized $24 \mathrm{~h}$ after ischemia. Protein levels of HSP70 and GRP78 in extracts from the striatum of the ipsilateral hemisphere were detected with immunoblotting. Levels of $\beta$-actin protein were used as the loading control. (A) The PGE1+Li mixture enhanced HSP70 protein expression. (B) The PGE1(S)+Li mixture enhanced GRP78 protein expression. The bar represents the mean \pm SD, $n=6$ rats. ${ }^{b} P<0.05$ compared with the sham-operated group; ${ }^{\mathrm{f}} P<0.01$ compared with the model group; ${ }^{\mathrm{h}} P<0.05$, i $P<0.01$ compared with the PGE1(S) group.

protein levels $(P<0.01$ vs model group and PGE1(S) group, Figure 5B).

\section{Discussion}

In a previous study, we found that coadministration of PGE1 (22.6 and $45.2 \mathrm{nmol} / \mathrm{kg}$, iv) and lithium $(0.5 \mathrm{mmol} / \mathrm{kg}$, sc) can induce substantial synergistic neuroprotection on focal cerebral ischemia compared with PGE1 or lithium alone. Thus, we formulated a PGE1+Li mixture (PGE1 $22.6 \mathrm{nmol} / \mathrm{kg}+\mathrm{Li} 0.5$ $\mathrm{mmol} / \mathrm{kg}$ or PGE1 $45.2 \mathrm{nmol} / \mathrm{kg}+\mathrm{Li} 0.5 \mathrm{mmol} / \mathrm{kg}$ ) for intrave-
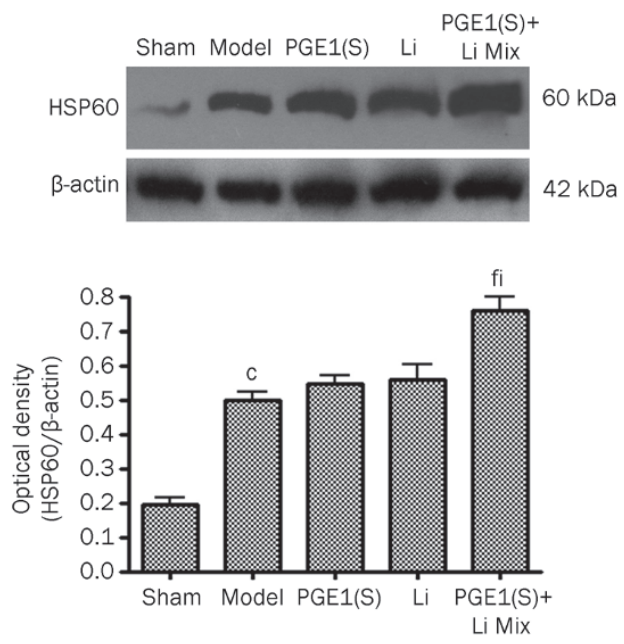

Figure 4. The PGE1+Li mixture enhanced pMCAO-induced HSP60 expression. The rats were injected intravenously with PGE1 22.6 nmol/kg, Li 0.5 mmol/kg or a PGE1(S)+Li mixture (PGE1 $22.6 \mathrm{nmol} / \mathrm{kg}+\mathrm{Li}$ $0.5 \mathrm{mmol} / \mathrm{kg}$ ) immediately after pMCAO. The rats were euthanized $24 \mathrm{~h}$ after ischemia. Protein levels of HSP60 in extracts from the striatum of the ipsilateral hemisphere were detected with immunoblotting. Levels of $\beta$-actin protein were used as the loading control. Bar represents mean $\pm S D, n=6$ rats. ${ }^{c} P<0.01$ compared with the sham-operated group; ${ }^{f} P<0.01$ compared with the model group; ${ }^{i} P<0.01$ compared with the PGE1(S) group.

nous administration. The results showed that the pMCAO rats had significant motor behavioral deficits and extensive infarction in the ipsilateral cerebral cortical and subcortical areas throughout a series of brain sections. In the groups treated with PGE1 or lithium alone, a reduction in infarct volume and improvement of neurological deficits was observed, but a greater reduction in infarct volume and neurological deficits was seen in rats that were given a PGE1(S)+Li mixture (PGE1 $22.6 \mathrm{nmol} / \mathrm{kg}+\mathrm{Li} 0.5 \mathrm{mmol} / \mathrm{kg}$ ). These results suggest that the PGE1+Li mixture might mimic the synergistic combination therapy of PGE1 and lithium reported in the previous study ${ }^{[14]}$. The suitable molar ratio of PGE1 to lithium for the PGE1+Li mixture was about $4.5 \times 10^{-5}: 1$. Moreover, the PGE1+Li mixture was effective even if it was administered $3 \mathrm{~h}$ after ischemia. Therefore, the PGE1+Li mixture might be an effective drug combination for the treatment of cerebral ischemia in clinical situations.

Previous work showed that neither PGE1 alone nor a combination of PGE1 with lithium influences the cerebrocortical blood flow of the pMCAO animals ${ }^{[14]}$. The results in this study were similar. When we monitored cerebrocortical blood flow after drug treatment, PGE1, lithium and the mixture of both compounds showed no significant improvements in the reduction of rCBF until $2 \mathrm{~h}$ after ischemia. Thus, the neuroprotection exerted by the PGE1+Li mixture in the rat brain ischemia model in this study could not be the result of increased cerebrocortical blood flow.

HSPs are molecular chaperones that bind to unfolded or 
A
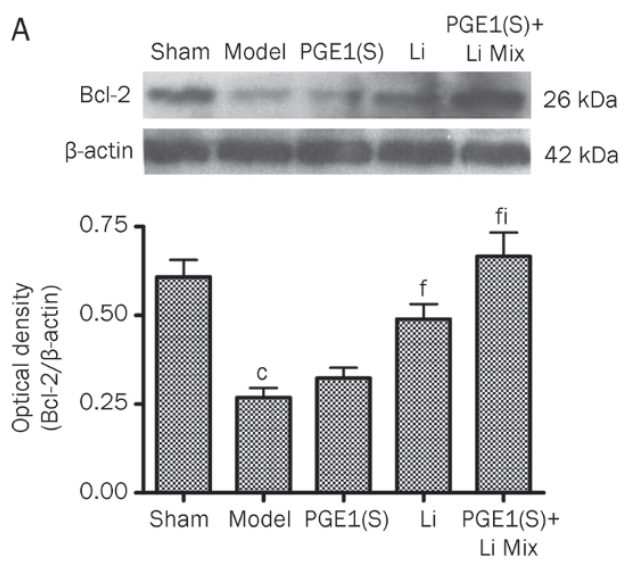

B
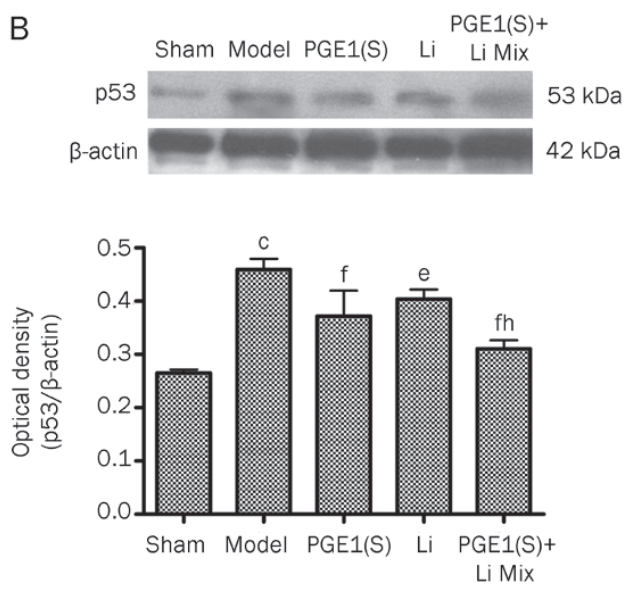

Figure 5. The PGE1+Li mixture increased $\mathrm{Bcl}-2$ protein expression, but decreased p53 protein expression. The rats were injected intravenously with PGE1(S) $22.6 \mathrm{nmol} / \mathrm{kg}$, Li $0.5 \mathrm{mmol} / \mathrm{kg}$ or the PGE1(S)+Li mixture (PGE1 $22.6 \mathrm{nmol} / \mathrm{kg}+\mathrm{Li} 0.5 \mathrm{mmol} / \mathrm{kg}$ ) immediately after pMCAO. The rats were euthanized $24 \mathrm{~h}$ after ischemia. Protein levels of $\mathrm{Bcl}-2$ and p53 in extracts from the striatum of the ipsilateral hemisphere were detected with immunoblotting. Levels of $\beta$-actin protein were used as the loading control. (A) The PGE1(S)+Li mixture increased Bcl-2 expression. (B) The PGE1(S)+Li mixture decreased p53 expression. The bar represents the mean \pm SD. $n=6$ rats. ${ }^{\circ} P<0.01$ compared with the sham-operated group; ${ }^{\mathrm{e}} P<0.05,{ }^{\mathrm{f}} P<0.01$ compared with the model group; ${ }^{\mathrm{h}} P<0.05,{ }^{\mathrm{i}} P<0.01$ compared with the PGE1(S) group.

misfolded proteins to ensure proper folding and prevent intracellular protein aggregation ${ }^{[2]}$. A number of studies have documented that HSP levels increase in the ischemic penumbra of the brain in animal models of focal ischemia, which is an area where many injured neurons survive ${ }^{[19]}$. It has been reported that gene transfer induced HSP70 overexpression protects neurons from ischemic brain damage in experimental rat stroke model ${ }^{[20]}$. Additionally, the overexpression of HSP70 inhibits the activation of NF- $\mathrm{kB}$, which is persistently activated during ischemia and appears to promote apoptotic cell death ${ }^{[21]}$. In contrast, the deletion of the HSP70 gene increases cytochrome $c$ release into the cytoplasm and subsequent caspase- 3 activation, thereby exacerbating apoptosis and increasing infarction volume after focal cerebral ischemia ${ }^{[22]}$. GRP78/Bip is one of the molecular chaperones localized to the ER membrane, which is also a highly conserved member of the 70-kDa heat shock protein family ${ }^{[8]}$. The main function of this protein is to restore folding of misfolded or incompletely assembled proteins. Previous reports showed that induction of GRP78 prevented the neuronal death induced by ER stress ${ }^{[23]}$, and BIX, a selective inducer of GRP78, could be used to prevent neuronal damage both in vitro and in vivo ${ }^{[24]}$. HSP60 is a mitochondrial matrix protein induced by stress that forms a chaperonin complex within the mitochondria, which is important for mitochondrial protein folding and function ${ }^{[9]}$. In prior studies, it was shown that induction of HSP60 protects neurons from ischemic damage ${ }^{[25]}$. In our ischemic model, HSP70, HSP60, and GRP78 protein levels significantly increased in the ischemic striatum after pMCAO. Although no significant increases in these HSPs were found when the animals were treated with PGE1 $22.6 \mathrm{nmol} / \mathrm{kg}$ or lithium $0.5 \mathrm{mmol} / \mathrm{kg}$ alone, the PGE1(S)+Li mixture robustly enhanced the increase in HSP70, GRP78, and HSP60 protein levels in the ischemic striatum. Therefore, induction of HSPs by the PGE1+Li mixture may play an important role in protecting neurons against ischemia-induced injury.

Overexpression of HSPs protects neurons against apoptotic and necrotic cell death during cerebral ischemia ${ }^{[4,26]}$. Thus, the combined treatment enhanced the changes in the expression levels of pro-apoptotic and antiapoptotic proteins. Recently, Bian reported that the neuroprotective effects of lithium were associated with a dramatic suppression in p53 expression as well as the upregulation of Bcl- $2^{[27]}$. Bcl-2 expression has been proposed as an important marker of the survival probability of neurons ${ }^{[28]}$. Bcl-2 has multiple neuroprotective actions including its ability to decrease Bax-induced cytochrome $c$ release from mitochondria and inhibit subsequent caspase activation $^{[29]}$. Lithium-induced upregulation of Bcl-2 was reported in cultured neurons and rat brains following protracted or short-term treatment ${ }^{[30]}$. The tumor suppressor protein $\mathrm{p} 53$, a transcriptional modulator of the $\mathrm{Bcl}-2$ genes, can produce cell cycle arrest and facilitate apoptosis ${ }^{[28]}$. The putative role of p53 in cerebral ischemia was based on observations that p53 is upregulated in the brain of rats subjected to focal ischemia ${ }^{[31]}$. Pifithrin- $a$, a p53 inhibitor, decreased the expression of p53targeted genes as well as the number of apoptotic cells in ischemic brains ${ }^{[32]}$. In the present study, the expression of p53 was significantly upregulated in the ischemic striatum accompanied by downregulation of Bcl-2. However, the PGE1+Li mixture significantly decreased p53 protein levels and increased Bcl-2 levels compared with the model group and the PGE1 group. These results suggest that the PGE1+Li mixture might protect neurons from cerebral ischemia by upregulating antiapoptotic proteins and downregulating pro-apoptotic proteins.

Although treatment with PGE1 or lithium alone reduced infarct volume, we did not find a significant increase in HSPs levels when the animals were treated with 22.6 or 45.2 $\mathrm{nmol} / \mathrm{kg}$ PGE1 or $0.5 \mathrm{mmol} / \mathrm{kg}$ lithium alone. The lack of 
effect on HSPs might be due to, in part, the low doses of lithium and PGE1 used in this study. Also, the effects on HSPs may have been too small to be detected with current methods. The alternative interpretation is that neuroprotection of PGE1 and lithium at these doses was not related to the induction of HSPs. It is possible that other molecular and cellular actions may participate in PGE1 and lithium-induced neuroprotection in the pMCAO model. These actions include the effects of PGE1 on cytoprotection, inhibiting platelet aggregation, membrane stabilization and anti-inflammation ${ }^{[10,33]}$, as well as the ability of lithium to inhibit $N$-methyl-D-aspartate receptors ${ }^{[34]}$, inhibit glycogen synthase kinase-3 (GSK-3) ${ }^{[35]}$, and induce expression of brain-derived neurotrophic factor in discrete brain areas ${ }^{[36]}$. The roles of other molecules in the neuroprotective effect caused by the PGE1+Li mixture in the ischemic model have yet to be defined. Nevertheless, the PGE1+Li mixture significantly enhanced the expression of HSP70, HSP60, and GRP78 in the ischemic striatum. These results suggest that induction of HSPs might take part in the molecular mechanisms of the neuroprotective effect of the PGE1+Li mixture against cerebral ischemia. However, it should be noted that these results can only provide indirect proof that the PGE1+Li mixture may exert protective effects on stroke through the induction of HSP proteins. Further study using HSP70 or GRP78 RNA silencing technology is needed to verify that the neuroprotective effect of the PGE1+Li mixture is actually mediated by HSPs.

In previous research from our laboratory ${ }^{[14,37]}$, lithium potentiated the neuroprotective effects of PGA1 and PGE1 through the upregulation of HSPs. However, PGA1 could only be delivered through intracerebral ventricle administration ${ }^{[37]}$, which is not a viable delivery route in clinics. Yet, the different administration routes of PGE1 (intravenous injection) and lithium (subcutaneous injection) made that drug combination inconvenient. In the present study, we prepared a combination of PGE1 and lithium that could be administered intravenously. This mixture produced synergistic neuroprotection against cerebral ischemia that was similar to the findings in our previous study. We believe that this PGE1+Li mixture has more practical application value, which might support a potential clinical therapy for ischemic cerebrovascular diseases.

In conclusion, we provided pharmacological evidence indicating that an intravenous PGE1+Li mixture was neuroprotective against cerebral ischemia. Reduction in injury volume was obtained even if the drug was administered $3 \mathrm{~h}$ after the induction of ischemia. The neuroprotective effect of the PGE1+Li mixture was associated with up-regulation of cytoprotective Bcl-2 and HSPs as well as down-regulation of the pro-apoptotic p53 protein in the ischemic brains. Our studies may provide a novel intravenous preparation for the clinical treatment of cerebrovascular diseases.

\section{Acknowledgements}

This work was supported by grants from the National Natural Science Foundation of China (№ 30801391 and 30930035), the Natural Science Foundation of Jiangsu Province (№ BK2007548), and the Social Progress and Medicine Foundation of Suzhou (No SS0729).

\section{Author contribution}

Zheng-hong QIN designed research and wrote the paper; Rui SHENG and Li-sa ZHANG performed research; Rui SHENG wrote the paper; Rong HAN corrected data; Bo GAO and Xiao-qian LIU analyzed data.

\section{References}

1 Onteniente B, Rasika S, Benchoua A, Guegan C. Molecular pathways in cerebral ischemia: cues to novel therapeutic strategies. Mol Neurobiol 2003; 27: 33-72.

2 Ohtsuka K, Suzuki T. Roles of molecular chaperones in the nervous system. Brain Res Bull 2000; 53: 141-6.

3 Yenari MA, Liu J, Zheng Z, Vexler ZS, Lee JE, Giffard RG. Antiapoptotic and anti-inflammatory mechanisms of heat-shock protein protection. Ann N Y Acad Sci 2005; 1053: 74-83.

4 Liu XQ, Sheng R, Qin ZH. The neuroprotective mechanism of brain ischemic preconditioning. Acta Pharmacol Sin 2009; 30: 1071-80.

5 Sheng R, Zhang LS, Han R, Liu XQ, Gao B, Qin ZH. Autophagy activation is associated with neuroprotection in a rat model of focal cerebral ischemic preconditioning. Autophagy 2010; 6: 482-94.

6 Tsuchiya D, Hong S, Matsumori Y, Shiina H, Kayama T, Swanson RA, et al. Overerexpression of rat heat shock protein 70 is associated with reduction of early mitochondrial cytochrome $c$ release and subsequent DNA fragmentation after permanent focal ischemia. J Cereb Blood Metab 2003; 23: 718-27.

7 Lee AS. The glucose-regulated proteins: stress induction and clinical applications. Trends Biochem Sci 2001; 26: 504-10.

8 Voos W, Rottgers K. Molecular chaperones as essential mediators of mitochondrial biogenesis. Biochim Biophys Acta 2002; 1592: 51-62.

9 Schror K, Hohlfeld T. Mechanisms of anti-ischemic action of prostaglandin E1 in peripheral arterial occlusive disease. Vasa 2004; 33: 119-24.

10 Matsuo K, Togo S, Sekido H, Morita T, Kamiyama M, Morioka D, et al. Pharmacologic preconditioning effects: prostaglandin E1 induces heat-shock proteins immediately after ischemia/reperfusion of the mouse liver. J Gastrointest Surg 2005; 9: 758-68.

11 Bauer M, Alda M, Priller J, Young LT. International group for the study of lithium treated patients (IGSLI). Implications of the neuroprotective effects of lithium for the treatment of bipolar and neurodegerative disorders. Pharmacopsychiatry 2003; 36: S250-4.

12 Chuang DM, Chen RW, Chalecka-Franaszek E, Ren M, Hashimoto R, Senatorov V, et al. Neuroprotective effects of lithium in cultured cells and animal models of diseases. Bipolar Disord 2002; 4: 129-36.

13 Ren M, Senatotov VV, Chen RW, Chuang DM. Postinsult treatment with lithium reduces brain damage and facilitates neurological recovery in a rat ischemia/reperfusion model. Proc Natl Acad Sci USA 2003; 100: 6210-5.

14 Han R, Gao B, Sheng R, Zhang LS, Zhang HL, Gu ZL, et al. Synergistic effects of prostaglandin E1 and lithium in a rat model of cerebral ischemia. Acta Pharmacol Sin 2008; 29: 1141-9.

15 Longa EZ, Weinstein PR, Carlson S, Cummins R. Reversible middle cerebral artery occlusion without craniectomy in rats. Stroke 1989; 20: 84-91.

16 Li F, Omae T, Fisher M. Spontaneous hyperthermia and its mechanism in the intraluminal suture middle cerebral artery occlusion model of rats. Stroke 1999; 30: 2464-71. 
17 Tamura A, Graham DI, McCulloch J, Teasdale GM. Focal cerebral ischemia in the rat: 1. Description of technique and early neuropathological consequences following middle cerebral artery occlusion. J Cereb Blood Flow Metab 1981; 1: 53-60.

18 Qin ZH, Chen RW, Wang Y, Nakai M, Chuang DM, Chase TN. Nuclear factor kappar B nuclear translocation upregulates c-Myc and p53 expression during NMDA receptor-mediated apoptosis in rat stratium. J Neurosci 1999; 19: 4023-33.

19 Wagstaff MJD, Collaco-Moraes Y, Aspey BS, Coffin RS, Harrison MJG, Latchman DS, et al. Focal cerebral ischaemia increases the levels of several classes of heat shock proteins and their corresponding $\mathrm{mR}$ NAs. Mole Brain Res 1996; 42: 236-44.

20 Li CY, Lee JS, Ko YG, Kim JI, Seo JS. Heat shock protein 70 inhibits apoptosis downstream of cytochrome $c$ release and up stream of caspase-3 activation. J Biol Chem 2000; 275: 25665-71.

21 Feinstein DL, Galea E, Aquino DA, Li GC, Xu H, Reis DJ. Heat shock protein 70 suppresses astroglial-inducible nitric-oxide synthase expression by decreasing NFkappaB activation. J Biol Chem 1996; 271 : 17724-32.

22 Lee SH, Kwon HM, Kim YJ, Lee KM, Kim M, Yoon BW. Effects of hsp70.1 gene knockout on the mitochondrial apoptotic pathway after focal cerebral ischemia. Stroke 2004; 35: 2195-9.

23 Reddy RK, Mao C, Baumeister P, Austin RC, Kaufman RJ, Lee AS. Endoplasmic reticulum chaperone protein GRP78 protects cells from apoptosis induced by topoisomerase inhibitors: role of ATP binding site in suppression of caspase-7 activation. J Biol Chem 2003; 278 : 20915-24.

24 Oidaa Y, Izutaa H, Oyagia A, Shimazawaa M, Kudob T, Imaizumic K, et al. Induction of BiP, an ER-resident protein, prevents the neuronal death induced by transient forebrain ischemia in gerbil. Brain Res 2008; 1208: 217-24.

25 Hwang IK, Ahn HC, Yoo KY, Lee JY, Suh HW, Kwon YG, et al. Changes in immunoreactivity of HSP6O and its neuroprotective effects in the gerbil hippocampal CA1 region induced by transient ischemia. Exp Neurol 2007; 208: 247-56.

26 Rajdev S, Hara K, Kokubo Y, Mestril R, Dillmann W, Weinstein PR, et al. Mice overexpressing rat heat shock protein 70 are protected against cerebral infarction. Ann Neurol 2000; 47: 782-91.

27 Bian Q, Shi T, Chuang DM, Qian Y. Lithium reduces ischemia-induced hippocampal CA1 damage and behavioral deficits in gerbils. Brain Res 2007; 1184: 270-6.

28 Li PF, Dietz R, von Harsdorf R. p53 regulates mitochondrial membrane potential through reactive oxygen species and induces cytochrome c-independent apoptosis blocked by bcl-2. EMBO J 1999; 21: $6027-$ 36.

29 Wada A, Yokoo H, Yanagita T, Kobayashi H. Lithium: potential therapeutics against acute brain injuries and chronic neurodegenerative diseases. J Pharmacol Sci 2005; 99: 307-21.

30 Chen RW, Chuang DM. Long-term lithium treatment suppresses p53 and Bax expression but increases Bcl-2 expression. J Biol Chem 1999; 274: 6039-42.

31 Watanabe H, Ohta S, Kumon Y, Sakaki S, Sakanaka M. Increase in p53 protein expression following cortical infarction in the spontaneously hypertensive rat. Brain Res 1999; 837: 38-45.

32 Leker RR, Aharonowiz M, Greig NH, Ovadia H. The role of p53-induced apoptosis in cerebral ischemia: effects of the p53 inhibitor pifithrin $\alpha$. Exp Neurol 2004; 187: 478-86.

33 Moncada S, Higgs EA. Prostaglandins in the pathogenesis and prevention of vascular disease. Blood Rev 1987; 1: 141-5.

34 Ma J, Zhang GY. Lithium reduced $N$-methyl- $D$-aspartate receptor subunit $2 \mathrm{~A}$ tyrosine phosphorylation and its interactions with Src and Fyn mediated by PSD-95 in rat hippocampus following cerebral ischemia. Neurosci Lett 2003; 348: 185-9.

35 Rowe MK, Chuang DM. Lithium neuroprotection: molecular mechanisms and clinical implications. Expert Rev Mol Med 2004; 6: 1-18.

36 Fukumoto T, Morinobu S, Okamoto Y, Kagaya A, Yamawaki S. Chronic lithium treatment increases the expression of brain-derived neurotrophic factor in the brain. Psychopharmacology 2001; 158: 100-6.

$37 \mathrm{Xu} \mathrm{XH}$, Zhang HL, Han R, Gu ZL, Qin ZH. Enhancement of neuroprotection and heat shock protein induction by combined prostaglandin A1 and lithium in rodent models of focal ischemia. Brain Res 2006; 1102: 154-62. 